\title{
Watch Try on using Augmented Reality
}

\author{
Prateek Singh $^{1}$ | Prof. Ajay Kaushik ${ }^{2}$ \\ ${ }^{1}$ B.Tech Student, Department of IT, Maharaja Agrasen Institute of Technology, Delhi, India \\ 2 Assistant Professor, Department of IT, Maharaja Agrasen Institute of Technology, Delhi, India
}

\section{To Cite this Article}

Prateek Singh and Prof. Ajay Kaushik, "Watch Try on using Augmented Reality", International Journal for Modern Trends in Science and Technology, 6(12): 418-420, 2020.

\section{Article Info}

Received on 16-November-2020, Revised on 09-December-2020, Accepted on 12-December-2020, Published on 18-December-2020.

\section{ABSTRACT}

In the ongoing occasions, Augmented Reality (AR) has been widely accepted as the new game changer for big tech companies. The idea of Augmented Reality is much past than creating gaming experiences like Pokemon Go. It is used to improve the experience of customers and provide excellent solutions for easily spotting manufacturing errors. During this paper, we will assess whether AR could be used for online shopping of Fashion Accessories and the future scope of the same. According to several studies conducted, around 20\% $40 \%$ of the garments sold by the online fashion retailer are returned by the customers. Out of this percentage, $60 \%-70 \%$ of the product returns happen due to sizing issues. Main reason to this inefficiency is wrong size ordered by the customer - mistakenly many times. Due to which, there is huge amount of money lost by a retailer simply due to extra shipping cost for returned items (to and fro) and it also leads to customer dissatisfaction.

KEYWORDS: Augmented Reality, Watch Try On, Vuforia SDK, Unity3D

\section{INTRODUCTION}

Augmented Reality is a technology that enhances the real world by affixing layers of digital elements onto it. These elements include computer-generated graphics, sound or video effects, haptic feedback, or sensory projects.

The intention behind adding this digital information is to provide an engaging and dynamic customer experience that is enabled with the input received from varied hardware like smart glass, smart lenses, and smartphones.

Augmented Reality (AR) is often mistaken with Virtual Reality (VR). The main difference between the two is that while Virtual Reality replaces the entire real environment with an artificial one, Augmented Reality is applied in a direct view of an existing real environment and adds elements like sounds, videos, or graphics onto it.
One of the most of consumed products of our time belongs to Fashion or Technology. So there was an obvious urge to combine

both and hence AR comes right at first - there seems to be a lot of potential in it.

Objective is to build a Marker-Based Augmented Reality experience which leverages the smartphone camera \& sensors capabilities to provide customers a try-on experience of fashion accessories (watches).

Build an interactive UI to change between 3D Models of Watches for trying them in real time and giving users the control to customise components such as color of the watch band. The paper is targeted towards the Fashion Industry to improve customer experience - both online $\&$ in-store. 
Augmented reality is the amalgamation of your real surrounding with augmented text or videos or graphics or GPS data created by computer, giving you an illusion of their presence in the real world. It differs from virtual reality as VR replaces the real environment with a simulated one whereas AR brings out the components of the digital world into a person's perceived real world.

Augmented reality can be incorporated into any device having hardware components like sensors, processors, displays and input devices. As modern day smart phones and tablets computers contain all these elements along with cameras and MEMS sensors, they are suitable platform for AR devices.

At present AR technology is rendered through an optical projection system, monitors, hand held devices and wearable display system may be HMD, eyeglasses, HUD, Contact lenses, Virtual retinal display and handheld.

Not so long ago augmented reality was a term relegated to the geekier corners of technology academia. But if you were to chart its public awareness, you would see a steep rise over the past 18 months that continues to build thanks to Google Glass and the many other companies that are stepping up to the challenge of commercializing it. Many people have an idea of what AR is, but few people have a vision for what it can be. Rather than attempt to define the term myself, I have set out to examine definitions offered by others:

Let's start simple with Mirriam Webster's definition: "An enhanced version of reality created by the use of technology to overlay digital information on an image of something being viewed through a device (as a smartphone camera); also : the technology used to create augmented reality.

\section{LITERATURE SURVEY}

Wang (2009) gives a point by point audit of AR in the AEC business, and gives a survey of a few significant exploration endeavors before 2009, and classifies different AR advances with their preferences and weaknesses.

Wang et al. (2013) audits 120 articles distributed somewhere in the range of 2005 and 2011 in different diary and gatherings information bases with an attention on expanded reality innovations in the fabricated climate. The paper arranges all accessible toolboxs for enlarged reality prototyping in five classes: 2D marker AR-PC and web-cam based, 2D marker AR-versatile, 3D article acknowledgment portable, marker-less apparatuses, GPS-compass based AR.

In their examination, AR writing is characterized in three classes: (1) application territory; (2) AR framework layers: idea and hypothesis (with four sub-layers including: calculation and displaying, reasonable structure, assessment system, and innovation selection), execution (with two sub-layers: programming and equipment), assessment (with two sub-layers: viability and ease of use), and industry appropriation; (3) other specialized measures. The paper investigates best in class innovations in every classification and proposes future exploration bearings.

Chi et al. (2013) talks about patterns in AR applications for the AEC/FM with a particular spotlight on four AR advances: confinement, normal UI, distributed computing, and cell phones. The paper surveys 101 articles and blueprints future patterns and open doors for applying AR in the AEC/FM industry in six bearings: (a) field investigation dependent on cross breed confinement, (b) in-field signal or sensation control of AR interface, (c) coordination with area explicit data, (d) getting to handle data utilizing omnipresent administrations, (e) convenient AR gadgets in the field, (f) setting mindful increased reality in AEC/FM fields.

\section{METHADOLOGY}

The development environment for the project is Unity Game Engine, Vuforia SDK and C\# language was used for programming the functionalities.

AR - a technology that superimposes a computer-generated objects on a user's view of the real world, thus providing a composite view.

It uses primary two components of a smartphone's camera: Gyroscope and Accelerometer to track the movement of the object in 3D space.

Together with reliable Software Development Kits and Engines, it creates an immersion - sense that digital objects belong in the real world.

Breaking immersion means that the sense of realism has been broken; in AR this is usually by an object behaving in a way that does not match our expectations. 


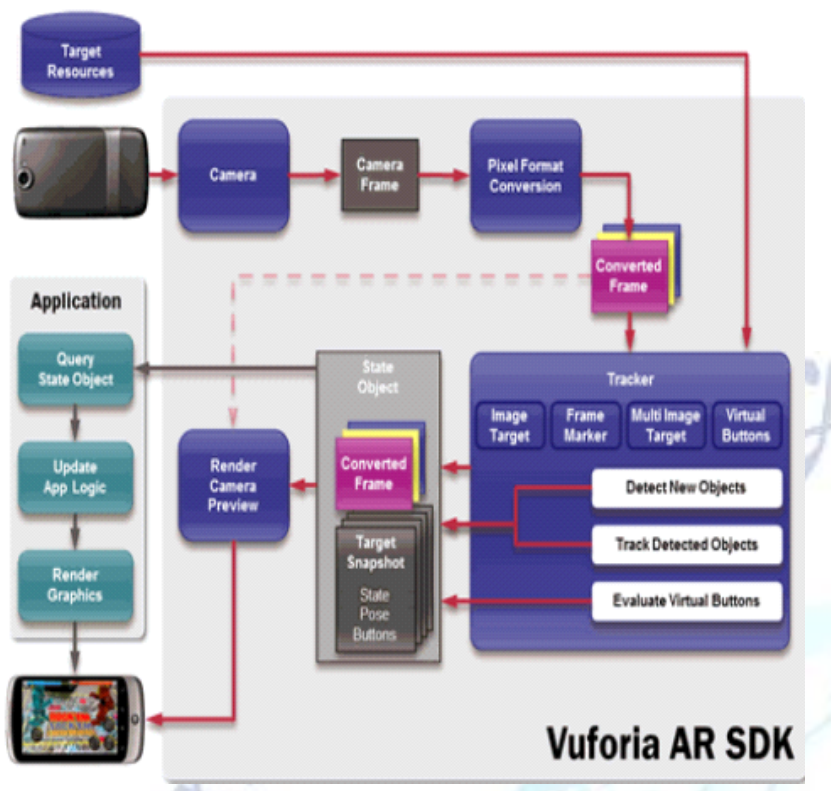

Image Target with visually distinct features is placed on user's wrist. Camera scans the image target.

Thereafter, it augments the 3D model of the Watch on top of that image and makes sure the back portion of the watch is occluded using another 3D model of the Hand/Wrist and applying Mask Shader on it.

Users can change the colors of the band and specifications by using the animated UI.

Switching between different models of watches is possible using C\# Scripting. Manual Testing is done to verify the functionalities.

\section{RESULTS}

We were able to produce a watch try on experience for the online shopping successfully and users get seamless experience while switching the 3D Models.

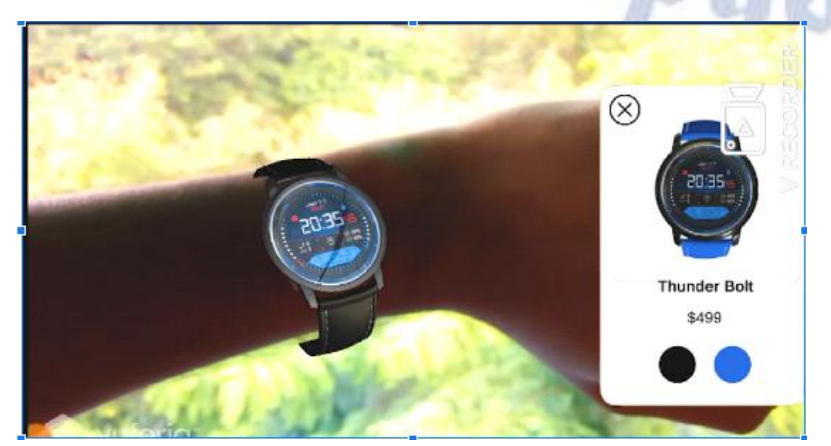

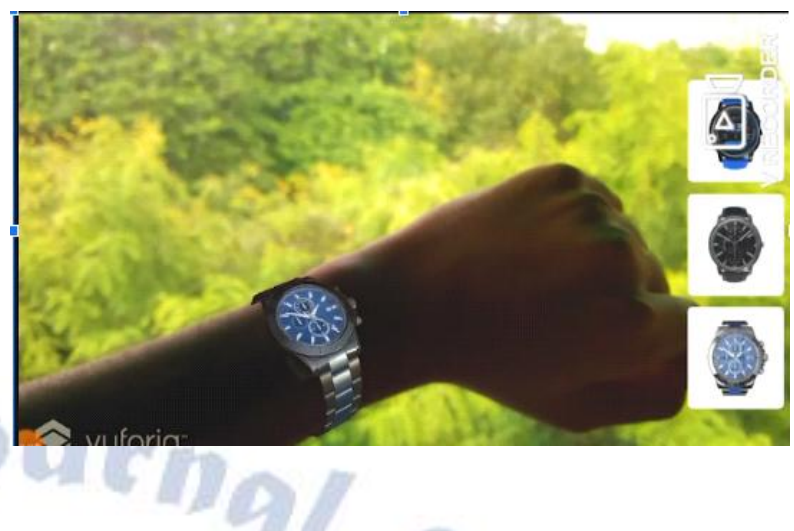

\section{CONCLUSION}

In this paper we have explained how to try on $3 D$ watch models using AR. We have explained the technologies and the methodology behind it. The upside of this system is the seamless experience throughout the cycle of trying out various items in AR environment. Unlike the traditional systems AR provides added advantages.

Currently, the designed system is confined to a simple application but this system can be used as the basis for a more complex AR system. We are currently using 3 models, these can also be replaced by an inventory.

This type of system can also be integerated into various online shopping stores and users can get a 'Try in AR' whenever they are using a mobile or any other such computable device.

So, it is safe to assume that the market share of AR is going to increase and in such times where a threatful virus has spread across the world, we realize the importance of online shopping and user experience on the platform.

\section{REFERENCES}

[1] NA Syed (2019). "Fashion Merchandising: an Augmented Reality", CUNY Academic Works.

[2] Anna Watson (2017). "Impact of Experential AR", Hertfordshire Business School, UK.

[3] Veronica Maccan (2016). "Impact of Technology in Fashion", Regent's University, London.

[4] Digit, Inside Issue Magazine - 19 (2018).

Hae Jung Suk (2014). "Using Unity3D to facilitate Augmented Reality Game Development”, Ajou University, Korea. 